\title{
What do I need to know about penicillin antibiotics?
}

Charlotte Barker (1, 2), Eva Germovsek (2), Mike Sharland (1)

(1) Paediatric Infectious Diseases Research Group, Institute for Infection and Immunity, St George's, University of London, London, UK; (2) Inflammation, Infection and Rheumatology section, Institute of Child Heath, University College London, London, UK.

\author{
Charlotte Barker \\ Paediatric Infectious Diseases Research Group \\ Institute for Infection and Immunity \\ St George's, University of London \\ Cranmer Terrace, London SW17 ORE, UK \\ Phone: 02087255382 \\ Fax: 02087250716 \\ E-mail: cisbark4444@doctors.org.uk \\ Eva Germovsek \\ UCL Institute of Child Health \\ Inflammation, Infection and Rheumatology section \\ 30 Guilford Street, London WC1N 1EH, UK \\ Phone: 02079052307 \\ Fax: 02079052882 \\ E-mail: eva.germovsek.11@ucl.ac.uk \\ Mike Sharland \\ Paediatric Infectious Diseases Research Group \\ Institute for Infection and Immunity \\ St George's, University of London \\ Cranmer Terrace, London SW17 ORE, UK \\ Phone: 02087255382 \\ Fax: 02087250716 \\ E-mail: mike.sharland@stgeorges.nhs.uk
}

Keywords: Beta-lactam, antimicrobial, paediatric, prescribe, pharmacology 


\section{What do I need to know about penicillin antibiotics?}

Penicillins belong to the beta-lactam family of antibiotics, which also includes carbapenems, monobactams, and cephalosporins. Penicillins are the class of antibiotics most frequently prescribed to children worldwide.[1] This paper summarises key principles of the pharmacology of penicillin antibiotics, which are clinically relevant when prescribing for children and infants.

\section{Mechanism of action}

Penicillins are bactericidal antibiotics that inhibit bacterial cell wall biosynthesis; pharmacologically, they target penicillin-binding proteins responsible for peptidoglycan cross-linking. Some bacteria display penicillin resistance; this can arise through several mechanisms, one of which involves drug inactivation by beta-lactamase enzymes; therefore penicillins may also be prescribed in combination with beta-lactamase inhibitors (BLIs) such as amoxicillin/clavulanate and piperacillin/tazobactam. The penicillins that are commonly prescribed for children are summarised in table 1.[2] 
Table 1. Summary of common penicillins with examples of indications and important factors for consideration when prescribing

\begin{tabular}{|c|c|c|c|c|}
\hline Type of penicillin & Example(s) & Spectrum & Common indications & Important factors \\
\hline \multirow[t]{3}{*}{ Natural penicillins } & \multirow[t]{2}{*}{$\begin{array}{l}\text { Benzylpenicillin sodium } \\
\text { (penicillin G) }\end{array}$} & \multirow[t]{3}{*}{ Narrow-spectrum } & $\begin{array}{l}\text { Early onset neonatal } \\
\text { sepsis (with gentamicin) }\end{array}$ & \multirow{2}{*}{$\begin{array}{l}\text { Penicillin } \mathrm{G} \text { is inactivated by gastric acid, so not suitable for oral } \\
\text { administration. } \\
\text { (Note benzylpenicillin should be available in community settings for } \\
\text { intramuscular administration in suspected meningococcal disease.) }\end{array}$} \\
\hline & & & $\begin{array}{l}\text { Suspected meningococcal } \\
\text { diseases (in community } \\
\text { setting) }\end{array}$ & \\
\hline & $\begin{array}{l}\text { Phenoxymethylpenicillin } \\
\text { (penicillin V) }\end{array}$ & & Streptococcal tonsillitis & $\begin{array}{l}\text { Suitable for streptococcal infection of mild-moderate severity. } \\
\text { Unpredictable absorption (from the gastrointestinal tract), so not suitable } \\
\text { for serious illnesses. Unpleasant taste. Four times daily dosing regimen } \\
\text { makes compliance difficult. }\end{array}$ \\
\hline \multirow[t]{2}{*}{ Antistaphylococcal } & Flucloxacillin & \multirow{2}{*}{$\begin{array}{l}\text { Penicillinase- } \\
\text { resistant }\end{array}$} & \multirow{2}{*}{$\begin{array}{l}\text { Skin and soft tissue } \\
\text { infections; osteomyelitis }\end{array}$} & \multirow{2}{*}{$\begin{array}{l}\text { Narrow spectrum: targets Gram-positive pathogens particularly } \\
\text { Staphylococcus aureus and Streptococcus pyogenes. Not suitable for MRSA } \\
\text { (methicillin-resistant Staphylococcus aureus) infections. Poor palatability. } \\
\text { Four times daily dosing affects compliance as above. }\end{array}$} \\
\hline & Oxacillin & & & \\
\hline \multirow[t]{2}{*}{ Aminopenicillins } & Amoxicillin & \multirow[t]{2}{*}{ Extended-spectrum } & $\begin{array}{l}\text { Amoxicillin is } \\
\text { recommended as first line } \\
\text { treatment for community } \\
\text { acquired pneumonia and } \\
\text { acute (bacterial) otitis } \\
\text { media. }\end{array}$ & $\begin{array}{l}\text { Semisynthetic penicillin. Amoxicillin has a similar antibacterial spectrum to } \\
\text { ampicillin but equivalent oral doses result in higher plasma concentrations } \\
\text { and it also has improved urinary excretion. Three times daily dosing may } \\
\text { improve compliance. }\end{array}$ \\
\hline & Ampicillin & & $\begin{array}{l}\text { Historical treatment of } \\
\text { choice for meningitis } \\
\text { caused by Listeria } \\
\text { monocytogenes. }\end{array}$ & $\begin{array}{l}\text { Semisynthetic, penicillin, which is closely related to amoxicillin. Ampicillin is } \\
\text { now rarely used in some countries (such as the UK) because its intravenous } \\
\text { formulation is very expensive, so few hospitals purchase it routinely. It } \\
\text { remains widely used elsewhere, such as in the US. Four times daily dosing } \\
\text { affects compliance as above. }\end{array}$ \\
\hline \multicolumn{5}{|c|}{ Combinations with beta-lactamase inhibitors (BLI) } \\
\hline (Aminopenicillin) & $\begin{array}{l}\text { Co-amoxiclav } \\
\text { (amoxicillin/clavulanic } \\
\text { acid) }\end{array}$ & Broad-spectrum & $\begin{array}{l}\text { Respiratory tract } \\
\text { infections (if amoxicillin } \\
\text { monotherapy not } \\
\text { appropriate), urinary tract } \\
\text { infections, animal bites }\end{array}$ & $\begin{array}{l}\text { Routinely prescribed as fixed dose combinations. Formulations suitable for } \\
\text { twice daily administration are available, which is beneficial for adherence. } \\
\text { Widely prescribed in emergency departments and general practice, with } \\
\text { negative implications for antimicrobial resistance (AMR). }\end{array}$ \\
\hline Ureidopenicillin & Piperacillin/tazobactam & Very broad & $\begin{array}{l}\text { Septicaemia, } \\
\text { febrile neutropaenia, }\end{array}$ & $\begin{array}{l}\text { Intravenous use only. Very broad spectrum. Increasingly prescribed in } \\
\text { hospital settings for serious infections such as neutropenic sepsis. Not yet }\end{array}$ \\
\hline
\end{tabular}




\begin{tabular}{|l|l|l|}
\hline & & $\begin{array}{l}\text { spectrum. } \\
\text { Antipseudomonal. }\end{array}$ \\
\cline { 1 - 2 } Carboxypenicillin & Ticarcillin/clavulanic acid &
\end{tabular}

Carboxypenicillin
Ticarcillin/clavulanic acid hospital-acquired

pneumonia

Septicaemia, bacteraemia

intra-abdominal sepsis licensed for use in neonates, although is used off-label in some neonatal

units.

Synergistic activity with aminoglycosides against Pseudomonas. Spectrum is not as broad as piperacillin/tazobactam. 


\section{Pharmacokinetics of the penicillins in children}

This section gives an overview of penicillin pharmacokinetics (PK): absorption, distribution, metabolism and elimination. The PK of penicillins is affected by their hydrophilic (water-soluble) nature.

(i) Absorption

Depending on the specific type of penicillin, absorption from the gastrointestinal tract can vary (see table 1). Absorption of certain penicillins, such as phenoxymethylpenicillin and flucloxacillin, is affected by the presence of food in the stomach, so patients and their parents should be advised that oral doses should be taken $30-60$ minutes before meals.

(ii) Distribution

The distribution of penicillins into most (non-specialist) tissues is usually excellent. In general, penicillin penetration of the blood-brain barrier is relatively poor (at approximately $20 \%$ )- and may be as low as $2 \%$ in the absence of meningeal inflammation. Therefore penicillins may only be used in the treatment of certain types of bacterial meningitis, such as intravenous benzylpenicillin for Group B Streptococcal meningitis in neonates. Of note, however, piperacillin/tazobactam has particularly poor central nervous system (CNS) penetration and is not the treatment of choice for CNS infections. There is usually no clinically relevant degree of plasma protein binding of penicillins, so the majority of the drug is free (unbound) and therefore pharmacologically active. While there is some evidence that this may change in critical illness, particularly for flucloxacillin, the data are as yet insufficient to alter routine care.

\section{(iii) Metabolism}

Hepatic metabolism is of very limited significance for penicillins. Some penicillins are metabolised in the liver to a degree and several metabolites have been identified including penicilloic acids. The unchanged drug and metabolites are excreted rapidly in the urine (see below) with only small amounts excreted in the bile.

\section{(iv) Elimination}

The penicillins are hydrophilic (water-soluble) compounds, which are principally renally excreted.[3] These drugs have a short half-life (e.g. 1.5 to 2 hours) in children with normal renal function. Concentrations achieved in the urine are usually sufficient to be therapeutic and these agents are therefore suitable for treatment of urinary-tract infections.[2] In critical illness, the half-life of penicillins may be prolonged due to impaired renal function. Conversely, critical illness may also induce augmented renal clearance, shortening the half-life of penicillins, by enhancing their elimination.[4] Therefore, in such circumstances, dose frequency may need to be reviewed, typically 
after consultation with either paediatric infectious diseases, microbiology, and/or specialist pharmacy teams.

If practising in the UK, when adjusting dose frequency (or dose) outside the recommendations of the British National Formulary for Children (BNFC [2]), specialist advice should be sought when possible. There is ongoing research into the potential role of therapeutic drug monitoring (TDM) for beta-lactam antibiotics in the critical care setting,[5] however this has not yet been introduced within routine paediatric intensive care.

\section{Issues specific to neonates}

The principal factors affecting neonatal penicillin pharmacokinetics are summarised in box 1.[3]

\section{Box 1. Neonatal physiological factors that influence penicillin pharmacokinetics.[3]}

- Increased body water content ( $75 \%$ of total body weight is water vs. $\sim 60 \%$ in adults) leads to increased volume of distribution and contributes to lowering circulating antibiotic concentrations (especially peak concentrations) in blood, requiring relatively high doses to treat severe infections such as meningitis.

- Relatively lower glomerular filtration rate and renal tubular secretion cause decreased penicillin clearance therefore dose frequency is reduced in the first weeks of life.

- Clearance further decreased in prematurity because of renal system immaturity, which prolongs penicillin half-life justifying further dose frequency reduction in preterm neonates.

- Renal function matures significantly within first days of life, increasing drug clearance and decreasing half-life, which requires increased dose frequency with increasing postnatal age (PNA).

In neonates, accurate assessment of renal function remains challenging, given the persistence of maternal creatinine for several days after birth: penicillin dosing regimens may warrant review if there is evidence of severe renal dysfunction (although dose alterations are rare in practice). There is very limited data regarding optimal penicillin dosing regimens in preterm neonates at varying gestational ages. As described in box 1, renal function matures rapidly with increasing PNA (thus decreasing penicillin half-life) but this is not currently reflected in most dosing guidelines, although some postmenstrual age based dosing regimens have been published. It is advisable to seek specialist guidance for complex cases when necessary. 


\section{Penicillin pharmacodynamics}

\section{Efficacy}

The efficacy of penicillin antibiotics is related to the percentage of time (within each dosing interval) that the concentration of the unbound fraction of the drug remains above the minimum inhibitory concentration (MIC) of the causative pathogen, generally abbreviated to \%T>MIC [6]. This concept is demonstrated in Figure 1 [7], together with the pharmacokinetic/pharmacodynamic (PKPD) indices that are important for other antibiotic classes [6].

This well-established adult pharmacokinetic/pharmacodynamic (PK/PD) index, \%T>MIC, is currently also the standard target of penicillin therapy for neonates and children. Historically, the target was to achieve at least $40 \% \mathrm{~T}>\mathrm{MIC}$ during therapy, and this is sometimes elevated to $70 \% \mathrm{~T}>\mathrm{MIC}$ (or higher) in critical illness. In practice, this is a difficult target to aim for, given that the majority of antimicrobial therapy is started empirically, in the absence of microbiologically confirmed infection. Even when positive cultures are obtained, it is not always possible to know the MIC of the responsible bacteria, and thus considering the MIC distributions of wild-type pathogen populations is important from the microbiological perspective. In paediatric antimicrobial therapy, empirical guidelines should consider the typical pathogens associated with particular conditions or infective syndromes in children, the likely MIC value(s), and local resistance profiles, and the principles of antimicrobial stewardship, which are discussed further below.[8]

\section{Toxicity}

Serious toxicity is rare in association with penicillin therapy. The most common adverse effect is antibiotic-associated diarrhoea (AAD), especially with broad-spectrum therapy such as co-amoxiclav. There is some evidence that different ratios of the amoxicillin to clavulanic acid components may affect the proportion of children who experience AAD.[9] Antimicrobial therapy can also rapidly alter the gut microbiome, which may contribute to $A A D$, although the longer-term effects are as yet unknown.

Other well-known adverse reactions to penicillins include drug-induced rash and hypersensitivity. Penicillin allergy is commonly reported but a thorough history surrounding the possible drug allergy is essential, particularly in critical illness where appropriate penicillin-based therapy can be lifesaving. If there is a known history of penicillin-related anaphylaxis, then caution should also be exerted with the use of certain other beta-lactam antibiotics, namely cephalosporins (especially first- and second- 
generation e.g. cefalexin, cefaclor) and carbapenems (e.g. meropenem), as cross-reactivity in the allergies between these classes can occur. Figures in the literature vary widely regarding how common cross-reactivity is, but its importance has frequently been overstated.[10] Penicillin allergy, of some type, is estimated to affect 1-10\% of people given penicillins[2]. On detailed investigation however, the true incidence in patients who claim a penicillin allergy is reportedly as low as $10 \%$.[10] In children likely to need recurrent penicillin-based therapy for serious illness (e.g. recurrent neutropenia in an oncological setting), a programme of desensitisation can be undertaken under specialist paediatric allergist/immunologist supervision, to overcome serious allergies.

Very rarely, seizures are reported in relation to penicillin therapy, and while extremely rare, this is an important consideration if higher than usual doses (or dose frequencies) are prescribed. Note that seizures have also been reported following rapid administration of high intravenous doses, therefore high doses should be infused slowly, over at least thirty minutes. Electrolyte imbalances can also arise secondary to penicillin administration, for example as penicillin sodium salts, which can contribute to hypernatraemia. This can be important in critical care, especially for neonates. The sodium content for some common penicillins in shown below in Table 2 .

Table 2. Sodium content of intravenous formulation of selected injectable penicillins (based on the Summaries of Product Characteristics (SPCS) available on the Electronic Medicines Compendium https://www.medicines.org.uk/emc/ )

\begin{tabular}{|l|r|}
\hline Penicillin (administered as sodium salt) & Sodium (mmol) / gram penicillin \\
\hline Amoxicillin & 2.60 \\
\hline Ampicillin & 2.73 \\
\hline Benzylpenicillin & 2.80 \\
\hline Flucloxacillin & 2.28 \\
\hline Piperacillin (with tazobactam sodium) & 2.36 \\
\hline Ticarcillin (with potassium clavulanate) & 4.37 \\
\hline
\end{tabular}

\section{Practical prescribing}

Paediatric penicillin dosing recommendations exist in various formats including age-bands, weightbased calculations $(\mathrm{mg} / \mathrm{kg}$ ), and weight-bands. There are strengths and limitations associated with each method of dose selection, discussed elsewhere.[11] The BNFC is the standard UK reference text for paediatric dosing[2] and in 2014, the dosing guidelines for oral amoxicillin and ampicillin were changed,[12] following concerns of widespread underdosing, especially in primary care.[13] Taking the specific example of amoxicillin, the standard recommended age-band doses for children were doubled (see Table 3). 
Table 3. Overview of BNFC changes to recommended standard oral doses of amoxicillin for children aged between 1 month and 12 years from April 2014

\begin{tabular}{|l|l|l|}
\hline Age-band & $\begin{array}{l}\text { BNFC dose recommendations } \\
\text { up to March 2014 }\end{array}$ & $\begin{array}{l}\text { BNFC recommendation from April } \\
\text { 2014 onwards }\end{array}$ \\
\hline Child 1 month-1 year & $62.5 \mathrm{mg} 3$ times daily & $125 \mathrm{mg} 3$ times daily \\
\hline Child 1-5 years & $125 \mathrm{mg} 3$ times daily & $250 \mathrm{mg} \mathrm{3} \mathrm{times} \mathrm{daily}$ \\
\hline Child 5-18 years & $250 \mathrm{mg} 3$ times daily & $500 \mathrm{mg} 3$ times daily \\
\hline
\end{tabular}

As new evidence from paediatric studies becomes available, dosing recommendations are thus regularly reviewed and updated, so it is helpful to use the online formulary resources when available, to access the latest evidence-based guidelines. Such changes also highlight the risks associated with old paperback formularies that may be kept on hospital wards, which may not reflect current dosing practices.

When prescribing oral penicillins, adherence is a major factor contributing to therapeutic success or failure. To maximise the chance of adherence to the proposed regimen, it is important to have a careful discussion with the child (providing they are old enough to understand) and their parent/carer, to communicate the proposed risks and benefits of the treatment regimen, and the importance of completing the antibiotic course. Practical strategies such as fitting in dosing times with the daily routine can be very important: twice daily regimens (avoiding a dose during school) are extremely helpful if clinically appropriate, when a suitable formulation is available. Avoiding four-times daily oral antibiotics (such as flucloxacillin) also warrants consideration, when clinically justifiable. For adherence, palatability is another aspect that should always be at the forefront of the prescriber's mind, as children are less likely to complete the course of an antibiotic which tastes unpleasant (e.g. flucloxacillin, and penicillin $V$ to a lesser extent) [14]. The difficulties parents may face in measuring the correct dose should also be borne in mind [15] and caregivers should be given guidance regarding how best to administer the dose to their child in an age-appropriate fashion. Consider also the shelflife of the prescribed medicine when choosing a course-length and discussing the plan with the family: for example, the reconstituted oral solution of penicillin $\vee$ has a shelf life of only seven days, meaning that parents would need to return to the pharmacy to collect a second bottle in order to complete a ten day course, which is unlikely to happen in practice. 


\section{Prescribing habits to avoid}

It is important to avoid excessive or inappropriate use of extended spectrum agents such as coamoxiclav in emergency department and general practice settings, which has been widely recognised. This is partly due to the common misconception that co-amoxiclav is suitable for penicillin-resistant pneumococcal infections. The reasons for curbing co-amoxiclav use are manifold, but include the longterm impact on antimicrobial resistance rates in the community, as well as possible patient-specific adverse effects (including $A A D$ and effects on the microbiome in the gut and beyond, possibly contributing to future diseases such as obesity [16]). Whenever possible, follow the main principles of paediatric antimicrobial stewardship.[8] Remember to include a review or stop date on every prescription to indicate the intended duration of therapy. Note, if your hospital uses electronic drug charts, beware that some software implements 'stop dates' automatically without informing the clinicians - so the antibiotic prescription suddenly disappears from the drug chart without requesting clinical review. Check whether this affects your local prescribing tool, and consider use of 'review dates' instead of 'stop dates' where appropriate.

\section{The future}

Although penicillins have been used for many decades in children and infants, many current paediatric dosing regimens were originally derived by scaling down adult dosing regimens using a linear weightbased $(\mathrm{mg} / \mathrm{kg}$ ) approach, which is now known to be suboptimal. $[6,17]$ This was because, historically, it was not feasible to do large scale paediatric antibiotic studies to verify the most effective dosing regimens for different indications; therefore, optimal antibiotic dosing for children is currently a highly active research area. As paediatric antibiotic PK/PD data improve, this will allow consolidation of the evidence to inform and update paediatric dosage regimens in formularies and SPCs.

Regarding PKPD targets of therapy, some experts now advocate aiming for $100 \%$ T>MIC by using continuous infusions, particularly in intensive care.[18] Given that many neonates receiving in-hospital antimicrobial therapy are either critically ill, or at risk of critical illness given their functional immunocompromise (immune system immaturity), some argue that we should also routinely aim for $70-100 \%$ T $>$ MIC in neonates. However, the evidence for continuous infusions is relatively sparse and this is another hot topic in antimicrobial research. Further, consideration must be given to the consequences of using continuous infusions such as (i) the potential need for additional intravenous access (ii) the volume required (iii) the chemical stability of the medicine itself during infusion (as penicillins are often unstable at room temperature), and (iv) the compatibility with other medications given through the same line. 


\section{Conclusions}

Globally, penicillins remain the most important group of antibiotics used to treat children and newborns. Appropriate usage is essential in the ongoing fight against antimicrobial resistance. When prescribing penicillins or indeed other antimicrobials for children, it is important to always apply the relevant principles of paediatric antimicrobial stewardship, which are summarised in box 2 .

\section{Box 2. Stewardship principles pertinent to penicillin prescribing in paediatrics}

1. Use a narrow(er)-spectrum penicillin whenever possible

2. Always set a completion or review date at the point of prescription

3. Switch from intravenous to orally administered antibiotics as early as feasible

4. Co-amoxiclav and other broader spectrum therapies are associated with higher rates of AAD, so may not be beneficial to your patient(s)

5. Concordance is paramount: discuss this explicitly with patients and their carers

Although co-amoxiclav is likely to remain popular among health professionals, remember to think critically before prescribing it and use amoxicillin instead unless you are specifically concerned about infection with amoxicillin-resistant bacteria such as Staphylococcus aureus or non-typeable Haemophilus influenzae. Always use the most up-to-date version of your formulary, as dosing recommendations do change, even for widely used antibiotics, as exemplified by amoxicillin. Improved paediatric penicillin PKPD data are awaited to optimize the evidence for modern dosing practices. In the meantime, it is recommended to follow your national formulary in conjunction with the relevant clinical guidelines when dosing penicillins for children, and to seek specialist advice when alternative dosing strategies are considered. 


\section{Learning point box: take home messages}

1. Penicillins are the class of antibiotics most frequently prescribed for children worldwide and they remain first-line therapy for a range of infections.

2. Penicillins are bactericidal antibiotics that inhibit bacterial cell wall biosynthesis by targeting penicillin binding proteins.

3. Penicillin antibiotics can be narrow-spectrum (e.g. benzylpenicillin) or extended-spectrum (e.g. amoxicillin), or broad-spectrum when combined with a beta-lactamase inhibitor such as amoxicillin/clavulanate (co-amoxiclav).

4. Penicillin antibiotics are principally renally excreted and the concentrations reached in the urine are adequate to be therapeutic for urinary-tract infections; penetration of the bloodbrain barrier is usually poor in the absence of inflammation.

5. Always take a thorough allergy history before prescribing antibiotics, but note that serious drug toxicity is rare with penicillins: the most common adverse effect is antibiotic-associated diarrhoea (AAD).

MCQs

1. Penicillin antibiotics target:

A. Bacterial cell wall synthesis

$T / F$

B. Bacterial DNA gyrase

C. Bacterial folic acid metabolism

$T / F$

D. Bacterial protein synthesis

$T / F$

$T / F$

2. Penicillin antibiotics:

A. are mainly metabolised in the liver $\quad T / F$

B. are mainly renally excreted $\quad T / F$

C. can be used in the treatment of urinary tract infections $\quad T / F$

D. penetrate well into the cerebrospinal fluid of healthy $T / F$ children

3. In critical illness, the clearance of penicillin antibiotics will be:

A. decreased because of impaired renal function

B. increased because of augmented renal clearance

C. A or B or neither

D. Neither A nor B

4. Penicillin allergy

A. is commonly reported by patients or their parents $\quad T / F$

$B$. is usually serious in nature $\quad T / F$

C. is always associated with allergy to cephalosporins $\quad T / F$

D. can be overcome with a specialist programme of $T / F$ desensitisation

\section{Contributorship statement}

$\mathrm{CB}, \mathrm{EG}$ and $\mathrm{MS}$ contributed to the preparation and review of this commissioned article. 


\section{Funding}

No specific funding was used for the preparation of this manuscript. C.I.S.B. is funded as a Clinical Research Fellow by the Global Research in Paediatrics Network of Excellence (GRiP), part of the European Union's Seventh Framework Programme for research, technological development and demonstration (FP7/2007-2013, Grant Agreement number 261060). E.G. is supported by an IMPACT PhD studentship from University College London (UCL), and has received funding from the neoMero study, part of the European Union Seventh Framework Programme for research, technological development and demonstration (Grant Agreement number 242146), and also from Action Medical Research (grant code SP4650, GN1834). M.S. chairs the UK Department of Health expert Advisory Committee on Antimicrobial Resistance and Healthcare Associated Infection, is an independent scientific advisor to NICE (The National Institute for Health and Care Excellence), and also receives institutional academic research grants from the NIHR (National Institute for Health Research) and the European Union.

\section{Competing interest}

The authors declare no competing interest.

\section{Acknowledgements}

The authors are grateful to Dr Joe Standing for comments on the manuscript.

\section{References}

1. Ahmed U, Spyridis N, Wong IC, et al. Dosing of oral penicillins in children: is big child=half an adult, small child=half a big child, baby=half a small child still the best we can do? BMJ 2011;343:d7803.

2. Paediatric Formulary Committee. BNF for Children (online) London: BMJ Group, Pharmaceutical Press, and RCPCH Publications http://www.evidence.nhs.uk/formulary/bnfc/current [Last accessed November 2015].

3. Pacifici GM, Labatia J, Mulla $\mathrm{H}$, et al. Clinical pharmacokinetics of penicillins in the neonate: a review of the literature. Eur J Clin Pharmacol 2009;65(2):191-8.

4. De Cock PA, Standing JF, Barker $\mathrm{Cl}$, et al. Augmented renal clearance implies a need for increased amoxicillin/clavulanic acid dosing in critically ill children. Antimicrob Agents Chemother 2015.

5. Roberts JA, Abdul-Aziz MH, Lipman J, et al. Individualised antibiotic dosing for patients who are critically ill: challenges and potential solutions. Lancet Infect Dis 2014;14(6):498-509. 
6. Barker $\mathrm{Cl}$, Standing JF, Turner MA, et al. Antibiotic dosing in children in Europe: can we grade the evidence from pharmacokinetic/pharmacodynamic studies - and when is enough data enough? Curr Opin Infect Dis 2012;25(3):235-42.

7. Connors KP KJ, Nicolau DP. Optimizing Antibiotic Pharmacodynamics for Clinical Practice. Pharmaceutica Analytica Acta 2013;4(3).

8. Bielicki J, Lundin R, Patel $S$, et al. Antimicrobial stewardship for neonates and children: a global approach. Pediatr Infect Dis J 2015;34(3):311-3.

9. Kuehn J, Ismael Z, Long PF, et al. Reported rates of diarrhea following oral penicillin therapy in pediatric clinical trials. J Pediatr Pharmacol Ther 2015;20(2):90-104.

10. Pichichero ME, Zagursky R. Penicillin and cephalosporin allergy. Ann Allergy Asthma Immunol 2014;112(5):404-12.

11. Bielicki JA, Barker $\mathrm{Cl}$, Saxena $\mathrm{S}$, et al. Not too little, not too much: problems of selecting oral antibiotic dose for children. BMJ 2015;351:h5447.

12. British National Formulary. April 2014 BNF e-newsletter. Available from http://www.pharmpress.com/mailouts/bnf/apr14/BNF enewsletter.html. [Last accessed November 2015].

13. Saxena S, Ismael Z, Murray ML, et al. Oral penicillin prescribing for children in the UK: a comparison with BNF for Children age-band recommendations. Br J Gen Pract 2014;64(621):e217-22.

14. Baguley D, Lim E, Bevan A, et al. Prescribing for children - taste and palatability affect adherence to antibiotics: a review. Arch Dis Child 2012;97(3):293-7.

15. Yin HS, Mendelsohn AL, Wolf MS, et al. Parents' medication administration errors: role of dosing instruments and health literacy. Arch Pediatr Adolesc Med 2010;164(2):181-6.

16. Principi N, Esposito S. Antibiotic administration and the development of obesity in children. Int J Antimicrob Agents 2016;47(3):171-7.

17. Anderson BJ, Holford NH. Understanding dosing: children are small adults, neonates are immature children. Arch Dis Child 2013;98(9):737-44.

18. Roberts JA, Lipman J, Blot S, et al. Better outcomes through continuous infusion of time-dependent antibiotics to critically ill patients? Curr Opin Crit Care 2008;14(4):390-6.

\section{Figure legend}

Figure 1. PKPD indices of antibiotic efficacy. $\mathrm{Cmax}=$ maximum concentration; $\mathrm{AUC} / \mathrm{MIC}=$ area under the curve / minimum inhibitory concentration; T>MIC = time above the MIC [7]. Reproduced under the terms of the Creative Commons Attribution License from Connors KP KJ, Nicolau DP. Optimizing Antibiotic Pharmacodynamics for Clinical Practice. Pharmaceutica Analytica Acta 2013; 4. 